\title{
OBESITY AND SLEEP-RELATED PATHOLOGY IN CHILDREN
}

\author{
Oana Iaru' ${ }^{1}$, Mihaela Oros ${ }^{2}$, D. Oraseanu ${ }^{1}$ \\ 'Pediatrics Department, ,, Grigore Alexandrescu“ Emergency Children's Hospital, \\ Bucharest \\ 2,,Medicover" Hospital, Bucharest
}

\begin{abstract}
Obesity is a public health problem, with an important increase in prevalence in the last decades, pediatric population also fits these characteristics. Among the systemic complications of obesity is included the sleep related respiratory pathology. The association obstructive sleep apnea - obesity is suspected in a child who snores, has sleep fragmentation and breathing pauses, but the diagnosis is confirmed using polysomnography. Obstructive sleep apnea has many kinds of complications (increased by the association with obesity) - cardiac, metabolic, neurocognitive, all of them affecting the quality of life. The treatment of obstructive sleep apnea includes weight loss, adenotonsillectomy and CPAP ventilation.
\end{abstract}

Keywords: obesity, obstructive sleep apnea

\section{CHILDHOOD OBESITY}

Obesity represented a real public health problem in the last decades because of its increased prevalence among both adult and pediatric population and also because of its long-term systemic consequences (cardiovascular, metabolic, renal, neurological, orthopedic, and psychosocial). According to data published by the National Health and Nutrition Examination Survey (comparative evaluation for the years 1976-1980 and 2003-2006), obesity prevalence increased significantly (increase from $5 \%$ to $12.4 \%$ for age group $2-5$ years, from $6.5 \%$ to $17 \%$ for age group 6-11 years and from 5\% to $17.6 \%$ for children between 12 and 19 years) (1).

To define obesity we have the CDC charts for body mass index: a body mass index above the 85 th percentile for age and sex is suggestive of an overweight child and a BMI above the 95th percentile means obesity.

Obesity can be regarded as a systemic involvement, with effects on the entire body. Among the complications associated with obesity a central role is occupied by the liver disease defined by two pathological entities: NAFLD (nonalcoholic fatty liver disease) and NASH (nonalcoholic steatohepatitis). The physiopathology of these hepatic diseases is characterised by local effects (steatosis, hepatocyte destruction, impaired liver function, inflammation) and systemic ones (increased cardiovascular risk, insulin resistance and type II diabetes, dyslipidemia). (2)

Metabolic syndrome is a clinical entity of its own, clearly defined in children over 6 years (not enough data to have a definition for children younger than 6 years) by the following criteria (IDF 2007): central obesity - waist circumference larger than $94 \mathrm{~cm}$ for boys and $80 \mathrm{~cm}$ for girls; triglycerides above $150 \mathrm{mg} / \mathrm{dl}$; HDL cholesterol less than $40 \mathrm{mg} / \mathrm{dl}$ in boys and $50 \mathrm{mg} / \mathrm{dl}$ in girls or specific treatment for dyslipidemia; blood pressure over $130 / 85 \mathrm{~mm} \mathrm{Hg}$ or treatment of previously diagnosed hypertension, fasting glucose level greater than $100 \mathrm{mg} / \mathrm{dl}$ or diabetes mellitus type II previously diagnosed. (3)

Other complications associated with obesity are: accelerated bone maturation, orthopedic pathology, hyperandrogenism, glomerulosclerosis, emotional and psychosocial problems. (4) 


\section{OBSTRUCTIVE SLEEP APNEA}

Regarding respiratory pathology associated with obesity, obstructive sleep apnea is characteristic. This syndrome has been described in children in 1970 and has the following definition: breathing disorder characterized by recurrent episodes of partial or complete upper airway obstruction, usually associated with intermittent hypoxemia and sleep fragmentation. (5)

The prevalence of obstructive sleep apnea is between 1 and $5.7 \%$, obesity being the most important risk factor for this condition (6), the peak incidence being encountered in the age group 5-10 years (11). The prevalence is equal in the boys girls groups, but there are also studies in which the proportion of boys is higher. (7)

Regarding the association between obstructive sleep apnea and obesity, the literature shows that approximately $10 \%$ of children diagnosed with obstructive sleep apnea are obese (8) and between 46 and $59 \%$ of obese children have respiratory sleep pathology on polysomnography. $(9,10)$; studies show that obesity also increases the risk of sleep apnea approximately 4 times.

From the pathophysiological point of view the obstructive sleep apnea syndrome has 4 phenotypes: the first one is characterized by adeno-tonsillar hypertrophy, it affects $2 \%$ of children between 2 and 8 years old and can cause severe cardiovascular and neurocognitive deficits if left untreated; the second one is associated with craniofacial malformations, is a syndromic phenotype (Down, PierreRobin) in which the respiratory impairment occurs soon after birth; the third phenotype is characterized by the association with primary neuromuscular disorders (Duchenne muscular dystrophy, spinal muscular atrophy) - can be associated with other respiratory changes (hypoxemia, hypoventilation without respiratory airways obstruction); the fourth phenotype is found in obese children.

\section{OBSTRUCTIVE SLEEP APNEA AND OBESITY}

In physiopathologycal terms, the association of these two entities can be explained by the interaction of anatomical factors (adenoid and tonsillar hypertrophy) and functional factors (increased airway collapsibility, chest wall mechanic alterations, decreased response to hypoxia and hypercapnia). Adeno-tonsillar hypertrophy (present in $45 \%$ of obese children with respiratory pathology associated) can be influenced by local or systemic inflammation, hormonal factors, the presence of other lo- cal structures - soft tissue - which can reduce the airway diameter. From another point of view, obesity decreases lung compliance and functional residual capacity, the consequences being: hypoventilation, increased work of breathing, ventilation/ perfusion mismatch. Obese children have also decreased ventilatory responses to hypoxia and hypercapnia.

\section{CLINICAL DIAGNOSTIC OF OBSTRUCTIVE SLEEP APNEA}

For infants and younger children the characteristic symptoms are: increased respiratory effort during sleep, snoring, breathing pauses/apnea, mouth breathing. They show abnormal positions during sleep (prone with the neck extended), and parents may complain of "sleep problems" such as: restless sleep, frequent movements, frequent awakenings. Children can have secondary enuresis and daytime fatigue, irritability

In older children the first sign is the presence of loud snoring; they may have also breathing difficulties, daytime sleepiness, impaired attention and concentration; morning headache may be associated occasionally.

\section{OSAS WORKUP}

The simplest method of diagnosis (but with low specificity) is an anamnestic one, by applying questionnaires to measure nocturnal respiratory symptoms. In this way we may identify some specific aspects (snoring), but respiratory pauses can't always be noticed by parents (mostly occur during REM sleep). Pediatric sleep questionnaire are few, mostly adaptations of the adults specific questionnaires; they have questions to show the respiratory symptoms during sleep, sleep quality, sleep pattern and daytime sleepiness.

Another diagnostic method is the nocturnal home oximetry, considered positive when the graph shows desaturations occurring during at least one period of 10-30 minutes. Positive oximetry may be specific for obstructive sleep apnea, but negative oximetry does not exclude the disease.

Another diagnostic method is respiratory polygraphy (overnight recordings of respiratory parameters). This technique does not allow monitoring of neurological parameters and sleep stages.

The gold standard for the diagnosis of obstructive sleep apnea is the polysomnography. With this technique we can assess the neurological (EEG, EMG) and respiratory parameters (nasal pressure, 
pulse oximetry, CO2 pressure). Obstructive Sleep Apnea is longer than two respiratory periods and occurs predominantly in REM sleep. OSAS is defined by the presence of apnea index greater than 1 and AHI (apnea - hypopnea index) greater than 1.5.

It should be mentioned that for younger children there is a different pattern of respiratory changes during sleep, they present significant upper airway obstruction during sleep, but without apneas, only with hypercapnia and/or hypoxemia; this pattern is called obstructive hypoventilation. (11)

\section{DIAGNOSTIC ALGORITHM FOR OBESE CHILDREN WITH POSSIBLE OBSTRUCTIVE SLEEP APNEA}

There are two main approaches for screening and diagnosis in children with these disorders: first we should have a proper diagnostic for overweight and obese children and for those we should apply the modified questionnaires validated for adult population with respiratory sleep disorders (Berlin questionnaire, Epworth sleepiness scale) and then perform another tests for the group of patients with positive questionnaires. The second approach is to make a proper diagnosis for the obese and nonobese children with sleep-related pathology (snoring, apnea, hypopnea, sleep fragmentation). (5)

\section{COMPLICATIONS OBESITY - OSAS}

\section{Cardiovascular}

As long-term effects of this combination we can talk about: pulmonary hypertension that can reach up to cor pulmonale, severe nocturnal hypoxemia due to hypercapnia and acidosis occurring during hypoventilation/apnea; we can also talk about systemic hypertension, left ventricular hypertrophy (left ventricular remodeling). (12)

In adults the association between obesity and sleep related respiratory pathology is clearly correlated with cardiovascular risk (acute coronary pathology, acute heart failure, stroke), as shown in a study realised on 6400 obese adults. (13) In children we don't have so many studies, but the literature also shows increased risk for systemic hypertension and loss of circadian modulation of blood pressure (lack of physiological nocturnal decline of blood pressure - nocturnal dipping). (14)

The pathophysiological mechanisms leading to these cardiovascular effects are: intermittent hypoxia and sleep fragmentation leading to activation of the sympathetic nervous system and increased oxidative stress, causing endothelial dysfunction and platelet activation.

\section{Metabolic}

Obstructive sleep apnea is a mediator for insulin resistance, dyslipidemia, inflammation, thrombosis through the following mechanisms: sympathetic discharge, intermittent hypoxemia, inflammation, thrombosis. Morbidity associated with these events is higher in children than in adults because of early onset.

There is an independent association of sleep respiratory disorders with insulin resistance (in the absence of obesity). (15)

The treatment of obstructive sleep apnea (adeno-tonsillectomy, CPAP) lowers the blood glucose level and the insulin resistance and determines a better functioning of pancreatic cells. The metabolic effects of the pathological association obesity OSAS are difficult to analyse because obesity by itself can have, alone, these consequences.

\section{Neurological}

The neurocognitive changes mentioned in the literature include: behavioral changes, learning difficulties, attention deficit, hyperactivity, aggressivity, decreased school performance.

Decreased school performance is determined by memory impairment and attention deficiency, learning skills damage; IQ of these children is lower compared with children of the same age who do not associate this pathology.

The neurocognitive changes can be explained by the following mechanisms: nocturnal hypoxemia due to repeated episodes of apnea/hypopnea causes cerebral ischemic injury, also sleep fragmentation and poor quality of sleep causes daytime somnolence, further contributing to the attention and concentration deficits.

Obese children with obstructive sleep apnea present memory deficits, poor vocabulary, speech disorders, learning difficulties, compared to obese children without respiratory pathology. (17)

Regarding the psychiatric pathology associated, a particular attention has to be accorded to ADHD, respiratory sleep disorders are highly predictive of long-term diagnosis of hyperactivity. (18)

Neuropsychological and cognitive deficits in children with respiratory pathology associated to sleep improve after treatment of this condition (improvement observed for hyperactivity, impulsivity, attention deficit); the quality of life of these children improves after treatment.

It is important to note that not all neuropsychological deficiencies improve with treatment, there 
are studies showing that language disorders and IQ are not significantly improved after treatment. (11)

\section{TREATMENT}

For the obese children with sleep-associated pathology the first therapeutic measure is weight loss (essential step that can often resolve the respiratory impairment or, if not, may be associated with other therapeutic measures).

Adeno-tonsillectomy is the treatment of choice for obstructive sleep apnea in children. As a complication of this therapeutic method is mentioned in the literature (greater frequency than in the general population) the appearance of laryngospasm. (19)

Studies have shown that the percentage of obese children with residual respiratory impairment after adeno-tonsillectomy is greater than the non-obese pediatric patients with the same treatment $(70 \%$ versus 30\%). Adeno-tonsillectomy improves AHI and oxygenation in obese children with obstructive sleep apnea, but $50 \%$ of them still have AHI (apnea index/hypopnea) greater than 5. (5) In conclusion we can talk about the improvement of obstructive sleep apnea after adeno-tonsillectomy in obese children, but with a higher percentage of residual disease if this therapeutic method is not associated with a program of weight loss.

Second line treatment is the use of CPAP ventilation (continuous positive airway pressure). Using CPAP is effective in the treatment of obstructive sleep apnea, but adherence to treatment is a significant problem,this is the reason why it is not recommended as first-line treatment.

Another treatment - positional therapy uses devices like shirts, pillows that promote sleeping in a adequate position to reduce obstructive sleep apnea in a subset of patients.

Uvulopalatopharingoplasty is a procedure in which the lateral pharyngeal pillars are trimmed and the uvula and posterior palate are excised to enlarge the respiratory airway, but after this procedure we can have significant complications - stenosis, dysphagia, velo-palatine insufficiency.

We can use also bariatric surgery for morbidly obese children, when other methods of weight loss have failed. According to studies, bariatric surgery significantly decreases respiratory symptoms associated with sleep apnea, but not totally, with lower CPAP requirements. (5).

\section{REFERENCES}

1. Ogden C.L., Flegal K.M. High body mass index for age among US children and adolescents 2003 - 2006, JAMA 299: 2401-2405, 2008

2. Pacifico L., Nobili V., Anania C. Pediatric NAFLD, metabolic syndrome and cardiovascular risk, World J. Gastroenterol. 17 (26): 3082-3091, 2011

3. www.idf.org (International Diabetes Federation guideline), 2007

4. Schwartz S. Obesity in children, Medscape

5. Arens R., Muzumdar H. Childhood obesity and obstructive sleep apnea syndrome, J. Appl Physiol, 108: 436-444, 2010

6. Redline S., Tishler P.V., Schluchter M., Aylor J., Clark K., Graham G. Risk factors for sleep-disordered breathing in children. Associations with obesity, race and respiratory problems, Am J Respir Crit Care Med, 159: 1527-1532, 1999

7. Marcus C.L., Brooks L.J., Draper K.A., Gozal D., Halbower A.C., Jones J., Schichter M.S., Ward S.D., Sheldon S.H., Shiffman R.N., Lehmann C., Spryt K. Diagnosis and management of childhood obstructive sleep apnea syndrome, Pediatrics, 130 (3): 714-755, 2012

8. Guilleminault C., Korobkin R., Winkle R. A review of 50 children with obstructive sleep apnea syndrome, Lung 159: 275-287, 1981

9. Marcus C.L., Curtis S., Koerner C.B., Joffe A., Serwint J.R., Loughlin G.M. Evaluation of pulmonary function and polysomnography in obese children and adolescents, Pediatr. Pulmonol., 21: 176-183, 1996

10. Silvestri J.M., Weese-Mayer D.E., Bass M.T., Kenny A.S., Hamptman S.A., Pearsall S.M. Polysomnography in obese children with a history of sleep-associated breathing disorders, Pediatr. Pulmonol, 16: 124-129, 1993

11. Trang H., Simonds A. Sleep disordered breathing in children, ERS Handbook, Respiratory Sleep Medicine, 205-209
12. Popescu V. Apneea obstructive in cursul somnului la copil, $R$ Rom Ped, 4(56): 350-358, 2007

13. Shahar E., Whitney C.W., Redline S., Lee E.T., Newman A.B., Javier Nieto F., O'Conner G.T., Boland L.L., Schwartz J.E., Samet J.M. Sleep-disordered breathing and cardiovascular disease: cross-sectional results of the Sleep Heart Health Study, Am J Resp Crit Care Med, 163: 19-25, 2001

14. Westerstahl E., Rossner S.M., Hedvall Kallerman P., Hagman E., Marcus C. Nocturnal blood pressure non-dipping is prevalent in severely obese, prepubertal and early pubertal children, Acta pediatr, 103 (2): 225-230, 2014

15. Redline S., Storfer-Isser A., Rossen C.L., Johnson N.L., Kirchner H.L., Emancipator J., Kibler A.M. Association between metabolic syndrome and sleep-disordered breathing in adolescents, Am J Respir Crit Care Med 176: 401-408, 2007

16. Harsch I.A., Schahin S.P., Radespiel Troger M., Weintz O., Jahariess H., Fuchs F.S. The effect of continuous positive airway pressure treatment on insulin sensitivity in patients with obstructive sleep apnea syndrome, Am J Respir Crit Care Med, 169: 156-162, 2004

17. Rhodes S.K., Shimoda K.C., Waid L.R. Neurocognitive deficits in morbidly obese children with obstructive sleep apnea, J Pediatr, 127: 741-747, 1995

18. Chervin R.D., Ruzicka D.L., Archbold K.H., Dillon J.E. Snoring predicts hyperactivity four years later, Sleep, 28(7): 885-890, 2005

19. Naffiu O.O., Prasad Y., Chimbira W.T. Association of childhood high body mass index and sleep disordered breathing with perioperative laryngospasm, Int J Pediatr Otorhinolaryngol, 77(12), 2044-2048, 2013 